\title{
ABELHAS EUGLOSSINAE DE BARREIRINHAS, ZONA DO LITORAL DA BAIXADA ORIENTAL MARANHENSE
}

\author{
José Manuel Macário REBÊLO', Antonio de Jesus M. CABRAL ${ }^{2}$
}

\begin{abstract}
RESUMO - Machos de Euglossinae foram coletados nos cerrados da região de Barreirinhas, Zona do Litoral da Baixada Oriental Maranhense, com uso de cineol, eugenol, salicilato de metila e benzoato de benzila, como iscas. Foram encontradas 9 espécies distribuidas em 4 gểneros: Euglossa, Eufinesea, Eulaema e Exaerete. As mais comuns foram Euglossa cordata (63,31\%), Eulaema cingulata (17,80\%), Eulaema nigrita (7,63\%), Euglossa modestior (4,94\%) e Eufriesea ornata $(4,16 \%)$. As demais, Euglossa chalybeata, Euglossa fimbriata, Euglossa melanotricha e Exaerete smaragdina, foram menos frequentes, representando juntas $2,16 \%$ da amostra total. As abelhas estiveram ativas nas duas estações, seca e chuvosa, sendo mais abundantes na última. Cineol atraiu $78,2 \%$ dos machos ( 8 espécies), eugenol, $19,9 \%$ (5 espécies), salicilato de metila 1,3\% (1 espécie) e benzoato de benzila $0,6 \%$ (2 espécies).
\end{abstract}

Palavras-chave: Abelhas, Euglossinae, iscas odoriferas, Maranhão

The Euglossine Bees of Barreirinhas, Western Coastal Lowland Zone of Maranhão State, Brazil

ABSTRACT - Male euglossine bees were collected in the savannas (Cerrado) of Barreirinhas, in the western coastal lowland zone of Maranhão State. Animals were baited with cineole, eugenol, methyl salicylate and benzyl benzoate. Nine species belonging to 4 genera were found. Euglossa cordata was the most abundant $(63,31 \%)$, followed by Eulaema cingulata (17,80\%), Eulaema nigrita (7,63\%), Euglossa modestior (4,94\%) and Eufriesea ornata $(4,16 \%)$. The others Euglossa chalybeata, Euglossa fimbriata, Euglossa melanotricha and Exaerete smaragdina, were less frequently found, representing only $2,16 \%$ of the total sample (combined). The bees were active in both the rains and dry seasons, especially on the first. Cineole was the most attractive aromatic compound $(78,2 \%$ of specimens and 8 species), followed by eugenol ( $19,9 \%$ and 5 species), benzyl benzoate $(0,6 \%$ and 2 species $)$ and methyl salicylate $(1,3 \%$ and 1 species).

Key-Words: Bees, Euglossinae, Chemical baits, Maranhão

\section{INTRODUÇÃO}

A subfamilia Euglossinae compreende um grupo de apjdeos exclusivamente neotropicais com a maior abundância de espécies ocorrendo nas zonas quentes úmidas equatoriais (MOURE, 1967).

E um dos poucos taxa de abelhas tropicais que mantêm relações restritas com grupos particulares de angiospermas, sem especificidade por pólen. As fêmeas são poliléticas e interagem nos trópicos, com outras abelhas de tamanho similar. Os machos por sua vez mantêm uma relação estreita com certas orquídeas numa síndrome baseada na oferta e coleta de compostos aromáticos (ACKERMAN, 1983), resultando daí a designação "as abelhas das orquideas". As substâncias aromáticas coletadas são utilizadas por eles na síntese de feromônios sexuais (WILLIAMS \& WHITTEN, 1983). Por esse motivo, se airaem facilmente por certos terpenóides e hidrocarbonetos aromáticos, sintéticos, análogos àqueles presentes nas fragrâncias florais.

1 Universidade Federal do Maranhào Departamento de Patologia Praça Madre Deus, $n^{\circ} 2 \mathrm{CEP}$ 65.025 - 560 São Luis - MA.

2 Auxiliar técnico. 
A utilização desses compostos como iscas tem auxiliado no conhecimento faunístico de diferentes áreas biogeográficas. No Brasil, a fauna de Euglossinae é melhor conhecida na região de Manaus, Amazônia Central (POWELL \& POWELL, 1987; BECKER et al., 1991; MORATO et al., 1992) e no nordeste do Estado de São Paulo (REBÊLO \& GARÓFALO, 1991; REBÊLO, 1993; MATEUS et al., 1993). Existem estudos também na Bahia (RAW, 1989; AGUILAR, 1990) e no Rio Grande do Sul (WITTMAN et al., 1988).

No Estado do Maranhão, com exceção de algumas espécies estudadas em São Luís (FERNANDES, 1991; GOMES, 1991; PEREIRA-MARTINS \& KERR, 1991), Vitória do Mearim (FERREIRA, 1994) e Alcântara (GONÇALVES et al., 1994), os Euglossinae são ainda desconhecidos em grande parte do seu território.

Com este trabalho, pretende-se iniciar a uma série de levantamentos de machos de Euglossinae, no Estado do Maranhão, começando pela Região da Baixada Oriental Maranhense.

\section{MATERIAL E MÉTODOS}

\section{Área de estudo}

O estudo foi realizado nos cerrados do Município de Barreirinhas, compreendido na Zona Litoral da Baixada Oriental Maranhense, tendo o ponto central localizado à $2^{\circ} 45^{\circ} \mathrm{S}$ e $42^{\circ}$ 5 ' W. A faixa litorânea do municipio é constituída pelos depósitos litorâneos marinhos e eólicos muito extensos e por aluviões flúvio-marinhos do Holoceno. Nesta área predominam as formações de cascalho, argila e areia (dunas), podendo-se encontrar em seus domínios restingas, mangues, campos de deflação. A zona sublitorânea e o interior pertencem ao grupo Barreiras, predominando os arenitos e em alguns trechos, areias finas (DNPM, 1973). A bacia de Barreirinhas é drenada pelo Rio Preguiças e seus afluentes. O municipio constitui o limite norte dos cerrados maranhenses que avançam do sul do Estado em direção ao litoral ocupando toda Bacia Parnaibana principalmente ao leste e ao sul. O cerrado naquela região é constituído por uma vegetação esparsa com plantas de períodos de floração alternados. Ali ainda se encontram manchas de mata margeando pequenos córregos que desaguam no Rio Preguiças e que vêm sofrendo acentuada ação antrópica - queimada/derrubada. O clima é do tipo sub-úmido megatérmico com precipitações anuais variando entre 2000 - 2200 mm (DNPM, 1973).

\section{Amostragens}

Machos de Euglossinae atraídos por iscas-odoríferas, foram capturados das 08:00 às 12:00 horas, duas vezes por mês, durante um ano (agosto de 1994 à setembro de 1995). Os compostos aromáticos usados para atrai-los foram cineol, eugenol, salicilato de metila e benzoato de benzila. $O$ método de captura das abelhas foi o mesmo empregado por REBÊLO \& GARÓFALO (1991). O estudo foi complementado por meio de registros de fềmeas quando em visitas às flores.

As abelhas coletadas neste estudo foram identificadas pelo primeiro autor e estão depositadas na coleção do setor de 
Entomologia do Departamento de Patologia da Universidade Federal do Maranhão-UFMA.

Dados climáticos, médios anuais, de temperatura, umidade relativa do ar e precipitação pluviométrica foram fornecidos pelo Instituto Nacional de Metereologia, Estação de Chapadinha-MA.

\section{RESULTADOS}

No total, foram encontradas 9 espécies de Euglossinae distribuídas por 4 gêneros: Euglossa ( $=$ Eg.), Eufriesea $(=E f)$, Eulaema $(=E l$.) e Exaerete (=Ex.). As mais comuns foram $\mathrm{Eg}$. (Euglossa) cordata (LINNAEUS), 1758 (63,31\%), El. (Apeulaema) cingulata (FABRICIUS, 1804) $(17,80 \%), \mathrm{El}$. (Apeulaema) nigrita Lepeletier, 1841 (7,63\%), Eg. (Euglossa) modestior DRESSLER, 1982c $(4,94 \%)$ e Ef. ornata (MOCSARY), 1896 (4.16\%). Enquanto Eg. (Glossura) chalybeata FRIESE 1925, Eg. (Euglossa) fimbriata
REBÊLO \& MOURE (no prelo), Eg. (Euglossa) melanotricha Moure, $1967 \mathrm{e}$ Ex. smaragdina (GUÉRINMÉNEVILLE, 1845) representaram juntas menos de $3 \%$ da amostra total (Tab.1).

Cineol foi o composto mais visitado, atraiu $78,2 \%$ dos machos e oito espécies. Na ordem de importância seguem eugenol (19,9\% dos machos e cinco espécies), salicilato de metila ( $1,3 \%$ dos machos e uma espécie) e benzoato de benzila $(0,6 \%$ dos machos e duas espécies) (Tab. 1 e Fig. 1)

Entre as nove espécies estudadas (tab. 1), quatro se atrairam por apenas uma isca: Eg. fimbriata, Eg. chalybeata e Eg. melanotricha foram atraidas por cineol e Ef. ornata por eugenol; quatro espécies visitaram duas iscas, sendo três delas mais frequentes na isca de cineol: Eg. cordata $(99,4 \%$ dos machos capturados), El, nigrita $(98,9 \%), E g$. modestior $(96,5 \%)$. Somente $E l$. cingulata

Tabela 1. Número de machos de Euglossinae atraídos por cineol $(=\mathrm{CI})$, eugenol $(=\mathrm{EG})$, salicilato de metíla $(=\mathrm{SM})$ e benzoato de benzila (=BB) em Barreirinhas, MA, Brasil, de outubro/94 à setembro/95.

\begin{tabular}{l|c|c|c|c|c|c}
\hline EUGLOSSINAE & CI & EG & SM & BB & TOTAL & $(\%)$ \\
\hline Eg. cordata & 726 & 4 & - & - & 730 & 63,31 \\
Eg. fimbriata & 3 & - & - & - & 3 & 0,26 \\
Eg. chalybeata & 17 & - & - & - & 17 & 1,47 \\
Eg. melanotricha & 2 & - & - & - & 2 & 0,17 \\
Eg. modestior & 55 & - & - & 2 & 57 & 4,94 \\
Ef. ornata & - & 48 & - & - & 48 & 4,16 \\
El. cinguata & 10 & 175 & 15 & 5 & 205 & 17,80 \\
El. nigrita & 87 & 1 & - & - & 88 & 7,63 \\
Ex. smaragdina & 2 & 1 & - & - & 3 & 0,26 \\
\hline TOTAL & 902 & 229 & 15 & 7 & 1153 & 100,0 \\
\hline
\end{tabular}




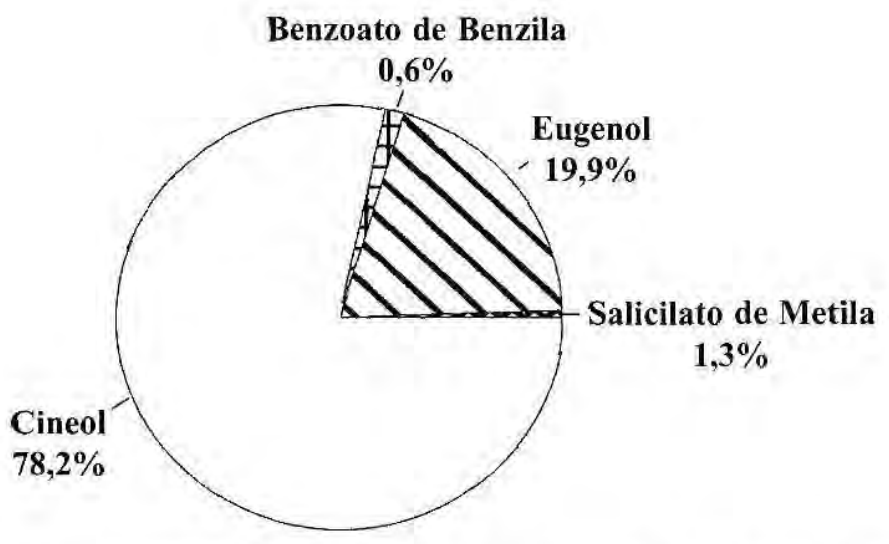

Figura 1. Número percentual de machos de Euglossinae atraidos por iscas de cineol, eugenol, salicilato de metila e benzoato de benzila, em Barreirinhas, MA, Brasil, de outubro/94 à setembro/95.

visitou todas as quatro iscas empregadas no estudo, sendo atraida com maior frequência por eugenol $(85,3 \%$ dos machos capturados).

Os machos visitaram as iscas nas duas estações do ano, estando presentes em todos os meses (Tab. 2). As maiores frequências de visitas ocorreram no período chuvoso, nos meses mais úmidos e também os de menores médias mensais de temperatura (Fig. 2). Nesta estação (dezembro à junho), foram registrados $71,6 \%$ dos machos capturados, contra
$28,4 \%$ nos meses secos (Fig. 3).

A maior concentração de espécies ocorreu na transição entre os períodos seco e chuvoso (novembro/dezembro) e no período chuvoso (março). Apenas Ef. ornata apresentou atividade sazonal restrita visitando as iscas somente nos meses de dezembro à março (Tab. 2).

\section{DISCUSSÃO}

A fauna existente na região de Barreirinhas é composto de elementos de diferentes padrões biogeográficos.

Tabela 2. Número de machos de Euglossinae atraidos por iscas-odoriferas de outubro/94 à setembro/95 em Barreirinhas, MA, Brasil.

\begin{tabular}{|c|c|c|c|c|c|c|c|c|c|c|c|c|c|}
\hline \multirow{3}{*}{$\begin{array}{l}\text { ANO } \\
\text { ESTAÇÃO } \\
\text { Euglossini/Meses }\end{array}$} & \multirow{2}{*}{\multicolumn{2}{|c|}{$\begin{array}{c}1994 \\
\text { SECA }\end{array}$}} & \multicolumn{10}{|c|}{1195} & \multirow{3}{*}{ TOTAL } \\
\hline & & & \multicolumn{7}{|c|}{ CHUVOSA } & \multicolumn{3}{|c|}{ SECA } & \\
\hline & 0 & $\mathrm{~N}$ & D & J & F & M & A & M & J & J & A & s & \\
\hline Eg cordala & 77 & 40 & 67 & 38 & 45 & 51 & 64 & 97 & 109 & $\$ 7$ & 49 & 36 & 730 \\
\hline Eg. fimbriata & 1 & & & & & 1 & & & & & & 1 & 3 \\
\hline Eg chalyheata & 1 & 1 & 1 & 4 & 1 & 2 & 4 & 2 & 1 & & & & 17 \\
\hline Eg. metanotricha & & 1 & & & & 1 & & & & & & & 2 \\
\hline Eg, modestior & 5 & 4 & 14 & 3 & 3 & 6 & 5 & 7 & 2 & & 3 & 5 & 57 \\
\hline Ef, ornata & & & 3 & 11 & 20 & 14 & & & & & & & 48 \\
\hline El. cingulata & 1 & 5 & 14 & 33 & 76 & 47 & 29 & & & & & & 205 \\
\hline El nigrita & 10 & 15 & 4 & 7 & 4 & 11 & 7 & 10 & 5 & 4 & 6 & 5 & 88 \\
\hline Ex. smaragdina & & & 2 & & & & 1 & & & & & & 3 \\
\hline$N^{9}$ de Espécies & 5 & 7 & 7 & 6 & 6 & 8 & 6 & 4 & 4 & 2 & 3 & 4 & \\
\hline $\mathrm{N}^{2}$ de Individuos & 94 & 67 & 105 & 96 & 149 & 133 & 110 & 116 & 117 & 61 & 58 & 47 & 1153 \\
\hline
\end{tabular}




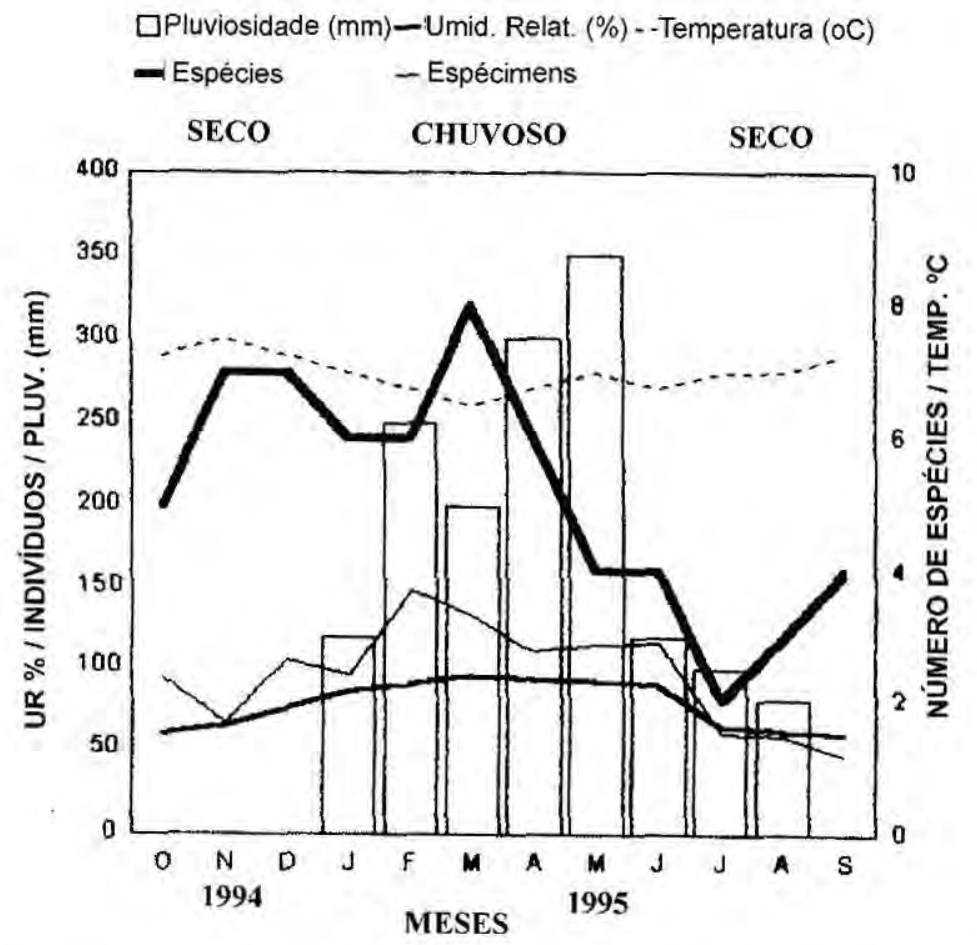

Figura 2. Fenologia dos machos de Euglossinae atraídos por iscas-odoriferas de outubro/94 à setembro/ 95, em Barreirinhas, MA, Brasil. Os dados referentes à temperatura estão multiplicados por 4.

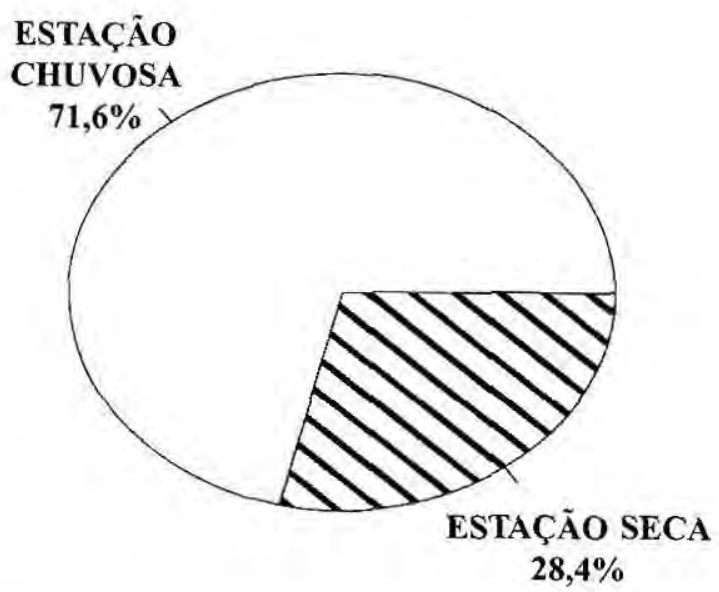

Figura 3. Número percentual de machos de Euglossinae atraidos por iscas-odoriferas nas estações seca e chuvosa, em Barreirinhas, MA, Brasil. 
A espécie predominante, Eg. cordata, tem ampla distribuição neotropical e é atraida muito facilmente por cineol e ocasionalmente por eugenol. Os machos podem ser encontrados o ano inteiro em ambientes diversos (cerrado, restinga, mata ciliar, mangue, campo, capoeira), sendo mais frequente nos meses de maio e junho. Eg. modestior também ocorre o ano inteiro. É uma espécie comum na Bacia Amazônica, embora seja encontrada também nas áreas xéricas de Minas Gerais (Obs. pes.). Foi atraida por benzoato de benzila e preferencialmente por cineol.

Menos comuns são Eg. melanotricha e Eg. fimbriata. Estas duas espécies são atraidas por cineol e foram registradas apenas no mês de novembro e março. Nas áreas subtropicais do Brasil, são atraídas por cineol, eugenol, e a última, também por vanilina em todas as estações, exceto no inverno, como tem se observado no Estado de São Paulo (REBÊLO \& GARÓFALO, 1991; REBÊLO, 1993). Ambas revelam-se mais frequentes nas savanas e matas semidecíduas do sul do Brasil (REBÊLO, 1993; REBÊLO \& GARÓFALO, 1991) do que nos trópicos úmidos (BECKER et al., 1991; GOMES, 1991; MORATO et al., 1992).

Eg. chalybeata embora pouco frequente em Barreirinhas, é comumente encontrada nas florestas da Bacia Amazônica, desde o Maranhão até o Peru, passando pelo Pará, Amazonas, Amapá, Guianas e Colombia (PEARSON \& DRESSLER, 1985; BECKER et al., 1991; GOMES, 1991; MORATO et al., 1992). É atraida por cineol, eugenol, acetato de benzila e salicilato de metila e ocorre também na Bolívia e no litoral de São Paulo e Paraná. Prefere ambientes de mata (FERNANDES, 1991).

Do gênero Eufriesea embora várias espécies sejam conhecidas para o norte do Maranhão, apenas Ef. ornata foi encontrada em Barreirinhas atraida frequentemente por eugenol, sendo este, o primeiro registro desta espécie para o Estado do Maranhão. Os registros prévios, no Brasil, indicavam sua ocorrência na Floresta Amazônica e na Mata Atlântica do Nordeste com virtual ausência no sertão (KIMSEY, 1982). É uma abelha de porte corporal avantajado, frequentemente confundida com as espécies do gênero Eulaema do complexo mimético encontrado na Bacia Amazônica e na Mata Atlântica (incluindo El. bombiformis, El meriana, El. seabrai), sendo facilmente separada destas pelo tergo e tíbia posterior dos machos enverdeados com tonalidades douradas devido à presença de pêlos amarelos (DRESSLER, 1979). É, como muitas espécies do gênero Eufriesea, sazonal, restringe sua atividade de campo à um período relativamente curto ( 4 meses), iniciando com as primeiras chuvas de dezembro permanecendo em atividade até o mês de maio. No Panamá tem sido registrada, também, apenas na estação úmida (ACKERMAN, 1983).

As duas espécies do gênero Eulaema, comuns na região de Barreirinhas, são de ampla distribuição neotropical: El. nigrita ocorre nas duas estações e em diversos ambientes: mata, cerrado, restinga, campo. Embora tenha a fama de preferir as áreas abertas e relativamente secas desde o Panamá até 
o Rio Grande do Sul, é muito comum nas matas tropicais de São Luís e São Paulo (REBÊLO \& GARÓFALO, 1991). É uma das poucas espécies que penetram nas caatingas nordestinas (AGUILAR, 1990). Se atrai por cineol o ano inteiro. El. cingulata prefere eugenol, mais também visita isca de cineol, salicilato de metila e benzoato de benzila. Em Barreirinhas foi muito comum de janeiro à março. Não foi coletado nenhum exemplar desta espécie de maio à setembro. Nas florestas tropicais do Panamá (ACKERMAN, 1983; ROUBIK \& ACKERMAN, 1987) ocorre em todas as estações.

Do gênero Exaerete tem-se encontrado, em Barreirinhas, machos de Ex. smaragdina sendo atraidos por isca de cineol e eugenol em dezembro e abril, e fêmeas de $E x$. dentata (Linnaeus, 1758) à procura de ninhos de hospedeiros. Machos desta última espécie não foram observados visitando isca. Ambas espécies apresentam ampla distribuição neotropical (KIMSEY, 1979).

\section{AGRADECIMENTO}

Os autores agradecem a Fundação de Amparo à Pesquisa no Maranhão (FAPEMA) pelo financiamento concedido.

\section{Bibliografia Citada}

ACKERMAN, J.D. 1983. Diversity and seasonality of male euglossinae bees ( $\mathrm{Hy}$ menoptera: Apidae) in Central Panamá. Ecology, 64:274-283.

AGUILAR, J.B.V. 1990. Contribuição ao conhecimento dos Euglossini ( $\mathrm{Hy}$ menoptera: Apidae) do Estado da Bahia, Brasil. São Paulo. Dissertação apresentada ao Mestrado de Ecologia do Instituto de Biociências da Universidade de São Paulo, SP, 96 p.

BECKER,P; MOURE, J.S.; PERALTA, F.J.A. 1991. More about euglossine bees in Amazonian Forest Fragments. Biotropica 23 (4b): 586-591.

DNPM - Projeto RADAM. 1973. Mapas de geologia e geomorfologia. Vol. 3: Folha SB-23 - Teresina e parte da Folha SB24 - Jaguaribe. Vol. 3: Folha SA - 23 São Luís e parte da Folha SA - 24 Fortaleza, Rio de Janeiro.

DRESSLER, R.L. 1979. Eulaema bombiformis, E. meriana, and mullerian mimicry in related species. Biotropica 11: 144-151.

FERNANDES, A. A. 1991. Avaliação do grau de preferência de abelhas Euglossini a ambientes com diferentes niveis de degradação. Monografia apresentada ao Curso de Ciências Biológicas da Universidade Federal do Maranhão, São Luís, MA, $51 \mathrm{p}$.

FERREIRA, R. da G. 1994. Estrutura de comunidades de abelhas (Hymenoptera, Apoidea) em uma regiâo de Baixada em Vitória do Mearim, MA, Brasil. Monografia apresentada ao Curso de Ciências Biológicas da Universidade Federal do Maranhão, São Luis, MA, $67 \mathrm{p}$.

GOMES, L.F. 1991. Diversidade e flutuação de populações de abelhas da tribo Euglossini (Hymenoptera, Apidae) em dois ecossistemas de São Luis: mata e restinga. Monografia apresentada ao Curso de Ciências Biológicas da Universidade Federal do Maranhão, São Luís, MA, $60 \mathrm{p}$.

GONÇALVES, S. de J. M.; RÊGO, M.; ARAÚJO, A. de. 1994. Abelhas sociais (Hymenoptera: Apidae) e seus recursos florais em uma região de mata secundária, Alcântara, MA, Brasil. Monografia apresentada ao Curso de Ciências Biológicas da Universidade Federal do Maranhão, São Luís, MA, (enviado para publicação).

KIMSEY, S.L. 1979. An illustrated key to the genus Exaerete with descriptions of male 
genitalia and biology. J. Kans. Ent. Soc, 52 : 735-746.

KIMSEY, S.L. 1982. Systematics of the genus Eufriesea. Univer. Calif. Publ. Entomol. 95: 1-125.

MATEUS, S; GARÓFALO, C.A.; REBÊLO, J.M.M. 1993. Espécies de Euglossini (Hymenoptera, Apidae) do Parque Estadual das Furnas do Bom Jesus, Pedregulho, SP. Anais do $14^{\circ}$ Congresso Brasileiro de Entomologia. Piracicaba, SP. p. 252.

MORATO, E.F; CAMPOS, L.A.; MOURE, J.S. 1992. Abelhas Euglossini (Hymenoptera, Apidae) coletadas na Amazônia Central. Rev Bras. Ent. 36 (4): 767-771.

MOURE, J.S. 1967. A check-list of the known euglossine bees (Hymenoptera, Apidae). Atas Simpos. Biota Amazôn., 5:395-415.

PEARSON, D.L.; DRESSLER, R.L, 1985. Two-year study of male orchid bee (Hymenoptera: Apidae: Euglossini) attraction to chemical baits in lowland south-eastem Peru. J. Tropical Ecol. 1: 37-54.

PEREIRA-MARTINS,S.R.; KERR,W.E. 1991. Biologia de Eulaema nigrita. 3. Inferências evolutivas. Papeis Avulsos Zool., 37 (15): 245-250.

POWELL, A.H.; POWELL, G.V.N. 1987. Population dynamics of male Euglossine bees in Amazonian Forest fragments. Biotropica. 19: 176-179.
RAW, A. 1989. The dispersal of euglossine bees between isolated patches of eastem Brazilian wet forest (Hymenoptera, Apidae, Euglossini). Rev. Bras. Ent. 33 (1): 103-107. REBÊLO, J. M. M. 1993. Dinâmica de populações de machos de Euglossinae (Hymenoptera: Apidae) e considerações sobre sua sistemática, filogenia e biogeografia. Tese de Doutorado apresentada ao Intituto de Biociência da Universidade Estadual Paulista, Rio Claro, SP, $168 \mathrm{p}$..

REBÊLO, J.M.M.; GARÓFALO, C.A. 1991. Diversidade e sazonalidade de machos de Euglossini (Hymenoptera: Apidae) e preferências por iscas-odores em um fragmento de floresta no sudeste do Brasil._Rev. Brasil, Biol., 51 (4): 787-799.

ROUBIK, D.W; ACKERMAN, J.D. 1987. Long-term ecology of euglossine orchidbees (Apidae: Euglossini) in Panama. Oecologia. 73: 321-333.

WILLIAMS, N.H.; WITTHEN, W.M. 1983. Orchid floral fragrances and male euglossine bees. Methodes and advances in the last sesquidecade. Biol. Bull., 164: 355-395.

WITTMAN,D; HOFFMANN, M.; SCHOLZ, E. 1988. Southern distributional limits of euglossine bees in Brazil linked to habitats of the Atlantic and Subtropical rain forest (Hymenoptera: Apidae: Euglossini). Entomol. Gener: 14 (1): 53-60. 